\title{
Infrared properties of planetary nebulae with [WR] central stars
}

\author{
S. K. Górny ${ }^{1}$, G. Stasińska ${ }^{2}$, R. Szczerba ${ }^{1}$, and R. Tylenda ${ }^{1}$ \\ 1 Copernicus Astronomical Centre, Department for Astrophysics, Rabiańska 8, 87-100 Toruń, Poland \\ 2 Observatoire de Paris-Meudon, DAEC, 92195 Meudon Cedex, France
}

Received 9 October 2000 / Accepted 16 July 2001

\begin{abstract}
We have gathered from the literature near and mid infrared (photometric and spectroscopic) data for Galactic planetary nebulae, with special attention to planetary nebulae surrounding Wolf-Rayet type central stars ([WR] PN). These data have been analyzed to obtain insight into the dust-properties of [WR] PN and their evolutionary status. We have found that a sizeable fraction of [WR] PN seems to contain hot dust (1000-2000 K), probably located in the winds of the central stars. The mean dust temperature is shown to decrease with decreasing $[\mathrm{WC}]$ spectral type. This is in line with suggestions that the [WC] sequence is an evolutionary sequence from late to early types. [WR] PN in different diagrams, when compared to those of non-[WR] PN, suggest that [WR] PN form a homogeneous class of planetary nebulae. There is an unusually large proportion of [WR] PN showing PAH features in their spectra.
\end{abstract}

Key words. planetary nebulae: general - stars: AGB and post-AGB - stars: Wolf-Rayet

\section{Introduction}

A phenomenon of planetary nebula is regarded as a transition phase in the evolution of low- and intermediatemass stars between the asymptotic giant branch (AGB) and a white dwarf configuration. Among more than a thousand planetary nebulae (PN) observed in our Galaxy, about 50 are known to have Wolf-Rayet type central stars ([WR] CS). They can easily be identified by their spectra which show numerous and strong stellar emission features and resemble those found in massive population I Wolf-Rayet stars of the class WC.

The emission spectra of ([WR] CS) are a manifestation of the very different nature of these stars from other nuclei of planetary nebulae. Detailed spectral analyses have shown [WR] CS to lose matter with typical rates of $10^{-6} M_{\odot} \mathrm{yr}^{-1}$ (e.g. Leuenhagen \& Hamann 1994, 1998; Leuenhagen et al. 1996; Koesterke \& Hamann 1997a; De Marco et al. 1997), that is, up to 2 orders of magnitude higher than other central stars (see e.g. Perinotto 1993). Their surface chemical composition is completely different from that of other PN central stars and comparable only to that of PG1159 stars (see e.g. Koesterke et al. 1998). As demonstrated by these analyses, the atmospheres of [WR] CS are mostly composed of helium and carbon and are almost completely hydrogen-free.

Send offprint requests to: S. K. Górny, e-mail: skg@ncac.torun.pl
Surprisingly, the pronounced differences between [WR]-type and normal central stars are not reflected in the nebulae themselves. The properties of PN surrounding [WR] CS do not seem different from those of other galactic planetary nebulae in most respects. In particular, this concerns chemical composition, morphology and electron densities (Górny \& Stasińska 1995, 1996; Górny 2001). The only prominent difference, higher expansion velocities of [WR] PN (Tylenda \& Górny 1993; Górny \& Stasińska 1995), can be attributed to an action of strong winds from the [WR] central stars.

It has been shown (Hu \& Dong 1992; Acker et al. 1996; Hamann 1997; Górny \& Tylenda 2000; Peña et al. 2001) that most [WR] PN seem to belong to an evolutionary sequence, from the late-type [WR] CS surrounded by compact nebulae to early-type [WR] CS surrounded by more diffuse nebulae. As showed by Górny \& Tylenda (2000) the most promising scenario for the creation of the majority of [WR] CS involves direct evolution from the AGB. Other evolutionary scenarios that have been proposed to explain the creation of [WR] CS assume a very late helium flash on the cooling track towards white dwarf stage (Iben et al. 1983; Schönberner \& Blöcker 1992) or evolution in a binary system that undergoes a common envelope phase (Tylenda \& Górny 1993).

In the present paper, we investigate the near and mid infrared properties of [WR] PN, which are indicative of their dust content and should give some additional clues 
Table 1. Infrared data for planetary nebulae with [WR]-type central stars.

\begin{tabular}{|c|c|c|c|c|c|c|c|c|c|c|c|c|}
\hline $\mathrm{PN} G$ & IRAS & name & spec. type & $E(B-V)$ & $(J-H)_{0}$ & $(H-K)_{0}$ & ref. & $\log F_{25} / F_{12}$ & $\log F_{60} / F_{25}$ & $T_{\mathrm{d}}$ & $m_{\mathrm{d}} / m_{\mathrm{g}} 10^{3}$ & class \\
\hline $000.4-01.9$ & $17512-2935$ & M 2-20 & [WC 6] & 1.07 & & & & & 0.21 & 93.25 & 3.55 & \\
\hline $001.5-06.7$ & $18129-3053$ & SwSt 1 & [WC 10] & 0.00 & 0.04 & 1.20 & $\mathrm{~b}, \mathrm{l}$ & 0.69 & -0.56 & 196.42 & 1.53 & D \\
\hline $002.4+05.8$ & $17262-2343$ & NGC 6369 & [WC 4] & 1.32 & -0.42 & 0.80 & $\mathrm{~d}, \mathrm{l}, \mathbf{n}$ & 0.86 & 0.22 & 92.06 & 2.35 & $\mathrm{H}$ \\
\hline $003.1+02.9$ & $17388-2440$ & $\mathrm{Hb} 4$ & [WC 3] & 1.13 & -0.90 & 0.92 & $\mathrm{f}$ & 0.88 & 0.31 & 87.10 & 4.83 & $\mathrm{E}$ \\
\hline $004.9+04.9$ & $17355-2206$ & M 1-25 & [WC 6] & 0.80 & & & & 0.91 & 0.13 & 98.70 & 5.09 & \\
\hline $006.8+04.1$ & $17425-2056$ & M 3-15 & [WC 5] & 1.26 & & & & 1.02 & 0.15 & 96.88 & 2.19 & \\
\hline $006.0-03.6$ & $18101-2530$ & M 2-31 & [WC 4-6] & 0.90 & & & & & 0.20 & 93.62 & 2.06 & \\
\hline $007.8-03.7$ & & M 2-34 & {$[\mathrm{WC}]$} & 0.93 & & & & & & & & \\
\hline $011.9+04.2$ & $17534-1628$ & M 1-32 & [WC 4] & 1.01: & & & & 0.58 & 0.06 & 103.82 & 3.29 & \\
\hline $012.2+04.9$ & $17514-1555$ & PM 1-188 & [WC 11] & & 1.44 & 2.06 & $\mathrm{i}, \mathrm{m}$ & 0.60 & -0.02 & 109.13 & & \\
\hline $017.9-04.8$ & $18383-1536$ & M 3-30 & [WC 2] & 0.71 & & & & & 0.37 & 83.57 & 0.21 & \\
\hline $019.4-05.3$ & $18430-1430$ & M 1-61 & [WC 4] & 0.63 & 0.54 & 0.01 & $\mathrm{~b}, \mathrm{l}$ & 1.08 & -0.19 & 128.22 & 4.11 & $\mathrm{R}$ \\
\hline $019.7-04.5$ & $18408-1347$ & M 1-60 & [WC 4] & 1.00 & & & & 1.04 & 0.18 & 94.99 & 7.78 & \\
\hline $020.9-01.1$ & 18307-1109 & M 1-51 & [WC 4-6] & $2.02:$ & & & & 0.81 & 0.13 & 98.12 & 1.76 & $\mathrm{H}$ \\
\hline $027.6+04.2$ & $18240-0244$ & M 2-43 & [WC 7] & $1.89:$ & -0.32 & 1.12 & c & 0.53 & -0.40 & 159.57 & 2.08: & $\mathrm{P}$ \\
\hline $029.2-05.9$ & $19032-0604$ & NGC 6751 & [WC 4] & 0.37 & -0.28 & 1.00 & $\mathrm{c}, \mathrm{n}$ & 0.67 & 0.11 & 99.97 & 4.29 & $\mathrm{H}$ \\
\hline $048.7+01.9$ & $19113+1454$ & He $2-429$ & [WC 4] & 1.34 & & & & 0.79 & 0.22 & 92.47 & 7.69 & \\
\hline $060.4+01.5$ & $19367+2458$ & HuDo 1 & [WC 11] & & & & & 0.61 & -0.14 & 121.85 & & \\
\hline $064.7+05.0$ & $19327+3024$ & $\mathrm{BD}+303639$ & [WC 9] & 0.23 & 0.06 & 0.93 & $\mathrm{~d}, \mathrm{n}$ & 0.42 & -0.16 & 123.78 & 18.74 & $\mathrm{P}$ \\
\hline $068.3-02.7$ & $20119+2924$ & He $2-459$ & [WC 9] & 1.34 & 0.31 & 0.93 & $\mathrm{j}$ & 0.92 & 0.02 & 106.93 & 9.86: & $\mathrm{L}$ \\
\hline $089.0+00.3$ & $21046+4739$ & NGC 7026 & [WC 3] & 0.57 & -0.51 & 0.73 & $\mathrm{~d}, \mathrm{n}$ & 0.89 & 0.37 & 83.84 & 4.41: & $\mathrm{E}$ \\
\hline $096.3+02.3$ & $21284+5414$ & K 3-61 & [WC 4-6] & $1.23:$ & & & & & 0.33 & 86.17 & 1.71: & \\
\hline $120.0+09.8$ & $00102+7214$ & NGC 40 & [WC 8 ] & 0.51 & & & & 0.70 & -0.05 & 112.19 & 0.99 & $\mathrm{~L}$ \\
\hline $130.2+01.3$ & $01539+6304$ & IC 1747 & [WC 4] & 0.59 & -0.38 & 0.68 & $\mathrm{~d}, \mathrm{n}$ & 0.74 & 0.34 & 85.59 & 3.09 & \\
\hline $144.5+06.5$ & $04026+6047$ & NGC 1501 & [WC 4] & 0.66 : & 0.06 & -0.02 & $\mathrm{n}$ & 0.66 & 0.44 & 80.51 & 1.56 & \\
\hline $146.7+07.6$ & $04215+6000$ & M 4-18 & [WC 11] & 0.47 & 0.58 & 1.10 & $\mathrm{j}$ & 0.35 & -0.38 & 154.91 & 1.48: & $\mathrm{R}$ \\
\hline $161.2-14.8$ & $03531+3343$ & IC 2003 & [WC 3] & 0.20 & -0.12 & 0.57 & $\mathrm{c}, \mathrm{d}, \mathrm{n}$ & 0.95 & -0.08 & 116.35 & $1.56:$ & \\
\hline $189.1+19.8$ & $07224+2935$ & NGC $2371-72$ & [WC 3] & 0.11 & -0.02 & 0.51 & $\mathrm{n}$ & 0.97 & 0.17 & 95.54 & 0.97: & \\
\hline $243.3-01.0$ & 07453-2712 & NGC 2452 & [WC 2] & 0.35 & -0.22 & -0.09 & c & 1.05 & 0.12 & 99.38 & 2.07 & \\
\hline $278.8+04.9$ & $10113-5005$ & PB 6 & [WC 2] & 0.42 & 0.06 & 0.22 & f & 0.81 & -0.07 & 115.46 & 2.02 & \\
\hline $278.1-05.9$ & $09200-5805$ & NGC 2867 & [WC 2] & 0.27 & -0.57 & 0.81 & c & 0.84 & 0.06 & 103.82 & 0.87 & $\mathrm{H}$ \\
\hline $285.4+01.5$ & $10364-5631$ & Pe 1- 1 & [WC 4-5] & 1.09 & & & & 1.00 & -0.19 & 127.75 & 4.63 & $\mathrm{H}$ \\
\hline $286.3+02.8$ & $10466-5547$ & He 2- 55 & [WC 3] & $0.41:$ & & & & & 0.02 & 107.31 & 0.51 & \\
\hline $291.3-26.2$ & $07027-7934$ & Vo 1 & [WC 11] & & 1.93 & 2.00 & $\mathrm{k}, \mathrm{l}, \mathrm{o}$ & 0.56 & -0.29 & 140.13 & $>10.96$ & $\mathrm{P}$ \\
\hline $307.2-03.4$ & $13300-6543$ & NGC 5189 & [WC 2] & 0.38 & & & & 1.03 & 0.40 & 82.21 & 0.43: & \\
\hline $309.0-04.2$ & $13487-6608$ & He 2- 99 & [WC 9] & 0.55 & -0.01 & 0.41 & $\mathrm{k}$ & 0.89 & 0.11 & 100.21 & 2.58: & \\
\hline $309.1-04.3$ & 13501-6616 & NGC 5315 & [WC 4] & 0.40 & 0.41 & -0.02 & c & 0.98 & 0.07 & 102.62 & 6.51: & $\mathrm{H}$ \\
\hline $321.0+03.9$ & $14562-5406$ & He $2-113$ & [WC 11] & 0.81 & 0.70 & 1.32 & $\mathrm{a}, \mathrm{c}, \mathrm{g}, \mathrm{e}, \mathrm{k}$ & 0.53 & -0.25 & 133.95 & $1.43:$ & $\mathrm{P}$ \\
\hline $332.9-09.9$ & $17047-5650$ & He $3-1333$ & [WC 11] & 0.58 & 0.76 & 1.64 & $\mathrm{a}, \mathrm{h}, \mathrm{k}, \mathrm{l}$ & 0.25 & -0.11 & 118.27 & $>56.55$ & $\mathrm{P}$ \\
\hline $336.2-06.9$ & $17023-5226$ & $\mathrm{PC} 14$ & [WC 4] & 0.48 & 0.74 & 0.19 & $\mathrm{f}$ & -0.16 & 0.23 & 92.43 & 1.81 & \\
\hline $337.4+01.6$ & $16268-4556$ & Pe $1-7$ & [WC 9] & 1.47 & 0.93 & 0.23 & $\mathrm{c}$ & 0.88 & -0.02 & 109.60 & 67.11: & $\mathrm{L}$ \\
\hline $352.9+11.4$ & $16416-2758$ & K 2-16 & [WC 11] & & -0.38 & 0.33 & $\mathrm{c}, \mathrm{l}$ & 0.60 & -0.10 & 117.01 & & $\mathrm{R}$ \\
\hline $355.2-02.5$ & 17409-3416 & Н 1-29 & [WC 4-6] & 0.87 & & & & & & & & \\
\hline $355.9-04.2$ & $17496-3437$ & M 1-30 & [WC 7] & 0.52 & & & & & 0.19 & 94.51 & 8.00 & \\
\hline $358.3-21.6$ & 19139-3942 & IC 1297 & [WC 3] & 0.15 & & & & 0.91 & 0.30 & 88.02 & 1.95 & \\
\hline
\end{tabular}

References: a) Webster \& Glass (1974); b) Allen \& Glass (1974); c) Persi et al. (1987); d) Peña \& Torres Peimbert (1987); e) Le Bertre et al. (1989); f) Preite-Martinez \& Persi (1989); g) van der Veen et al. (1988); h) Gaylard et al. (1989);

i) Garcia-Lario et al. (1990); j) Zhang \& Kwok (1990); k) Zijlstra et al. (1991); l) Fouqué et al. (1992); m) Hu et al. (1993);

n) Phillips \& Cuesta (1994); o) Garcia-Lario et al. (1997).

to their formation and evolution. In Sect. 2 we present the observational samples and the data sources. In Sect. 3, we discuss the near infrared properties of $[\mathrm{WR}] \mathrm{PN}$, and in Sects. 4 and 5 the mid infrared photometric and spectroscopic properties, respectively. The main conclusions are summarized in Sect. 6 .

\section{The PN samples and observational data}

The [WR] PN sample is the same as in Górny \& Tylenda (2000). It contains all the [WR] PN known in our Galaxy, based on the lists compiled by Tylenda et al. (1993) and Acker et al. (1999). This sample comprises only objects that are certainly planetary nebulae and have central stars that can be at least approximately classified using similar criteria to the ones used for genuine, massive population I
Wolf-Rayet stars of class WC. We rejected PN for which the $[\mathrm{WR}]$ nature of the central star is uncertain as well as all PN with so-called weak emission line central stars (see Tylenda et al. 1993). The PN G identifications, IRAS numbers and usual names of the $[\mathrm{WR}] \mathrm{PN}$ are given in Cols. 1 to 3 of Table 1 .

The spectral type of each [WR] CS has been adopted from the literature and is given in Col. 4.

The basic nebular and stellar data, nebular $\mathrm{H} \beta$ fluxes, densities and diameters, stellar $V$ magnitudes, reddening and surface brightness $\left(S_{\mathrm{H} \beta}\right)$ are taken from the same sources or calculated in the same way as in Górny \& Tylenda (2000) and are listed in Table 1 of that paper.

The near infrared photometry has been compiled from the literature and is based mostly on inputs to the Strasbourg-ESO Catalogue of Galactic Planetary Nebulae 
(Acker et al. 1992) and to the Catalog of Infrared Observations (Gezari et al. 1997). The adopted values of the reddening-corrected $(J-H)_{0}$ and $(H-K)_{0}$ colours for each $[\mathrm{WR}] \mathrm{PN}$ are given in Cols. 6 and 7 of Table 1. A complete list of references is given in Col. 8. When there are several references in the literature for one object, we took for each colour the simple mean of values calculated from data given by different authors. The fluxes have been dereddened using the reddening law of Rieke \& Lebofsky (1985). The colour excesses $E(B-V)$ are given in Col. 5 of Table 1 .

The IRAS flux densities have been adopted from the Strasbourg-ESO Catalogue of Galactic Planetary Nebulae (Acker et al. 1992) and in a few cases supplemented with data from the University of Calgary IRAS Database. The colours calculated from IRAS fluxes are given in Cols. 9, 10 of Table 1 . The dust temperature $T_{\mathrm{d}}$ and the dust-to-gas mass ratio $m_{\mathrm{d}} / m_{\mathrm{g}}$ have been calculated with the same method as in Stasińska \& Szczerba (1999). They are listed in Cols. 11 and 12 of Table 1. The IRAS LRS spectral classes from Volk \& Cohen (1990) are given in Col. 13.

The control sample is composed of the non-[WR] planetary nebulae from the Strasbourg-ESO Catalogue of Galactic Planetary Nebulae (Acker et al. 1992). For those objects, we used the near infrared and IRAS data from that catalogue. Their near infrared fluxes have been reddening-corrected with $E(B-V)$ calculated as in Górny \& Tylenda (2000) and data adopted from sources listed therein.

\section{Near infrared photometry}

Near infrared photometric observations can reveal what the dominant source of radiation is at this wavelength. Colour-colour diagrams are especially useful in this respect and have frequently been used in the past (e.g. Whitelock 1985; Persi et al. 1987). Figure 1 presents the $(H-K)_{0}$ vs. $(J-H)_{0}$ colour-colour diagram. [WR] PN are represented by pentagons and non-[WR] PN by open circles. For reference purposes, we have also drawn several lines. The thick solid line represents the intrinsic colour indices of main sequence stars (Koorneef 1983), the bottom corresponding to B-type stars, the top to M-type stars. The dotted line represents the colour indices of free-free and bound-free radiation from plasmas of different temperatures (Persi et al. 1987) and the dashed line represents dust colour indices for temperatures $T_{\mathrm{d}}<2000 \mathrm{~K}$ and emissivity exponent 0 (Phillips \& Cuesta 1994). The relative location of the sources with respect to these lines allows one to guess what the main contributor to the nearinfrared radiation is: nebular radiation $(\mathrm{N})$, hot dust (D) or stellar continuum (S).

As can be seen from Fig. 1, the distributions of $[\mathrm{WR}] \mathrm{PN}$ and non-[WR]PN are different in this plot. While most of the non-[WR] PN occupy a rather limited zone, sometimes referred to as the "nebular box"

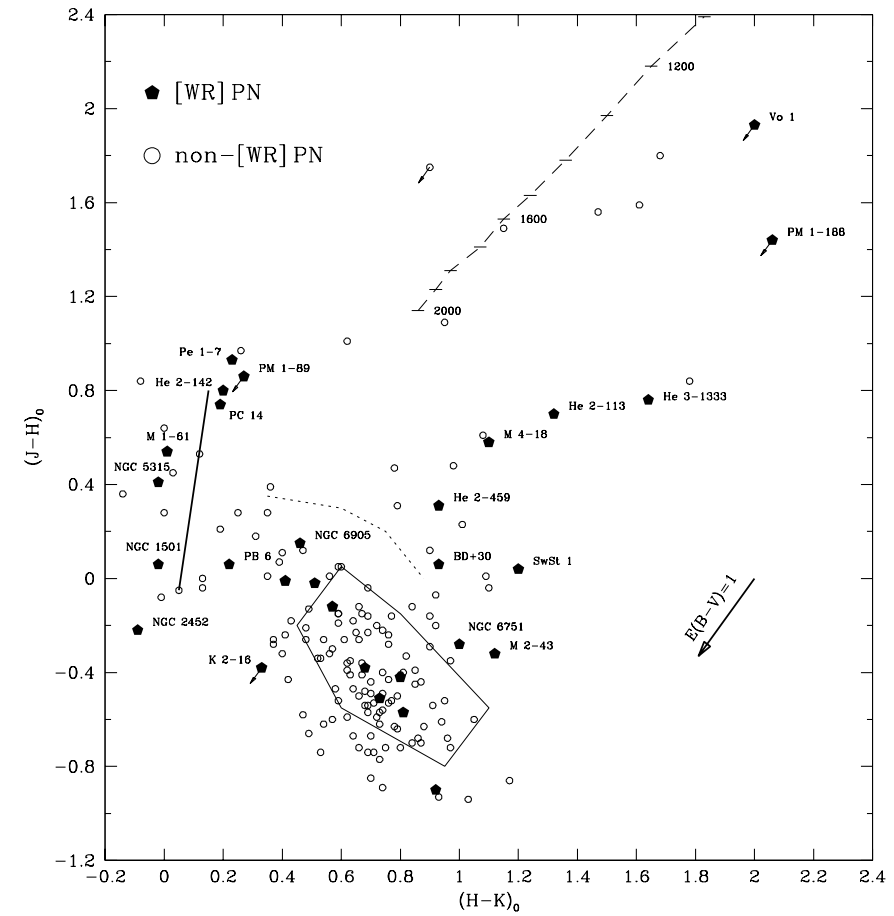

Fig. 1. Colour-colour diagram for $[\mathrm{WR}] \mathrm{PN}$ (pentagons) and other PN (open circles). The solid line stands for the intrinsic colours of main sequence stars (Koorneef 1983). The dotted line represents free-free and bound-free radiation from plasmas of different temperatures (Persi et al. 1987). The dashed line represents dust colour indices for temperatures $T_{\mathrm{d}}<2000 \mathrm{~K}$ and emissivity exponent 0 (Phillips \& Cuesta 1994).

(Whitelock 1985), the [WR] PN are spread over a much larger surface in the plot.

About $30 \%$ of the [WR] PN are found close to the stellar main sequence line, while only a small proportion of non- $[\mathrm{WR}] \mathrm{PN}$ are found in that zone. Let us first note that the $[\mathrm{WR}] \mathrm{PN}$ central stars that lie close to the main sequence line have [WR] types between [WC 9] (He 2-142 and Pe 1-7) and [WC 2] (PB 6 and NGC 2452), so that their effective temperatures range between $5 \times 10^{4} \mathrm{~K}-$ $1.4 \times 10^{5} \mathrm{~K}$ (Leuenhagen et al. 1996; Koesterke \& Hamann $1997 \mathrm{~b})$, i.e. they are much higher than the temperatures of main sequence stars. Obviously their near infrared colours do not reflect those of the central stars atmospheres. The fact that there is a large proportion of $[\mathrm{WR}] \mathrm{PN}$ in the main sequence zone as compared to non-[WR] PN may just be a result of our selection of the observational data. For $[\mathrm{WR}] \mathrm{PN}$, we have extensively searched the literature and taken the observed values as specified in Sect. 2 while for non-[WR] PN, we have simply taken the near infrared fluxes as they appear in the Strasbourg-ESO catalogue. Actually, in the colour-colour diagram of Persi et al. (1987), there are many PN lying close to the main sequence star region, whose near infrared data do not appear in the Strasbourg ESO catalogue, possibly because they were suspected of contamination by field stars. PreiteMartinez \& Persi (1989) have reobserved 14 sources classified as S by Persi et al. (1987) and found 11 of them to 
be in fact of type $\mathrm{N}$ but this was never proved to be the case for all the remaining $\mathrm{PN}$.

Near infrared photometric observations were typically done with a $15^{\prime \prime}$ aperture and can easily be contaminated by neighbouring stars, especially in crowded fields. This was suspected for PC 14 by Preite-Martinez \& Persi (1989) and could be the case also for other our objects. Indeed, we have checked that for most [WR] PN with infrared colours resembling main sequence stars, many field stars are present nearby and especially for Pe1-7 and PM 1-89, contamination from another star is very probable. Still, some of the [WR] PN with colour indices of main sequence stars could have binary nuclei with one component being precisely a main sequence star. So far, observational campaigns carried out in the visual wavelengths (e.g. by Ciardullo et al. 1999 for NGC 1501 and a few other [WR] PN) have not detected binary central stars in any of the [WR] PN. Still, the hypothesis of the binary nature of the nine [WR]-type central stars lying close to the main sequence region is worth investigating because it could have important consequences for our understanding of the evolution of these objects. As we have already recalled in the introduction, the binary scenario is one of the possible evolutionary paths for [WR] PN.

Only about $40 \%$ of the $[\mathrm{WR}] \mathrm{PN}$ lie in or near the "nebular box" and probably have near-infrared radiation dominated by the nebular component. They are located below the plasma radiation line, possibly because of an excess in the $J$ band caused by contribution from nebular emision. The main contributor is the helium triplet at $1.083 \mu \mathrm{m}$ (Whitelock 1985) but important contributions from different lines to the $J$ and $K$ bands are also possible (Phillips \& Cuesta 1994).

About $30 \%$ of the $[\mathrm{WR}] \mathrm{PN}$ (namely: $\mathrm{BD}+30^{\circ} 3639$, He 2-459, SwSt 1, M 4-18, He 2-113, He 3-1333, PM 1-188 and Vo 1) are located in a zone of the diagram where only few of the non-[WR] PN are found. Their colours can be explained by a contribution from hot dust (1000-2000 K) as already suggested by Zijlstra et al. (1991). This dust would arise in the wind of the [WR] nucleus. Note that all these $[\mathrm{WR}] \mathrm{PN}$ have late ([WC 9] - [WC 11]) spectral type nuclei with relatively slow $\left(\sim 300 \mathrm{~km} \mathrm{~s}^{-1}\right.$, Leuenhagen et al. 1996, 1998) and presumably dense winds. On the other hand, $\mathrm{K} 2-16$ is also a PN with a late type ([WC 11]) central star but hot dust seems to play no important role in this case.

It is interesting to compare, in the $(H-K)_{0}$ vs. $(J-H)_{0}$ plane, the location of these late-type $[\mathrm{WR}] \mathrm{PN}$ with late-type population I WC stars. This is done in Fig. 2 where the population I WC stars are taken from Williams et al. (1987). For the latter objects, the near infrared colours have been interpreted in terms of hot dust emission in the winds of these stars (Williams et al. 1987) and models for the formation of carbon grains at distances of about 500-1000 stellar radii have been proposed (Cherchneff \& Tielens 1995; Zubko 1998). Since the atmospheres of population I and population II Wolf-Rayet stars of the same subtype are homologous (the spectral

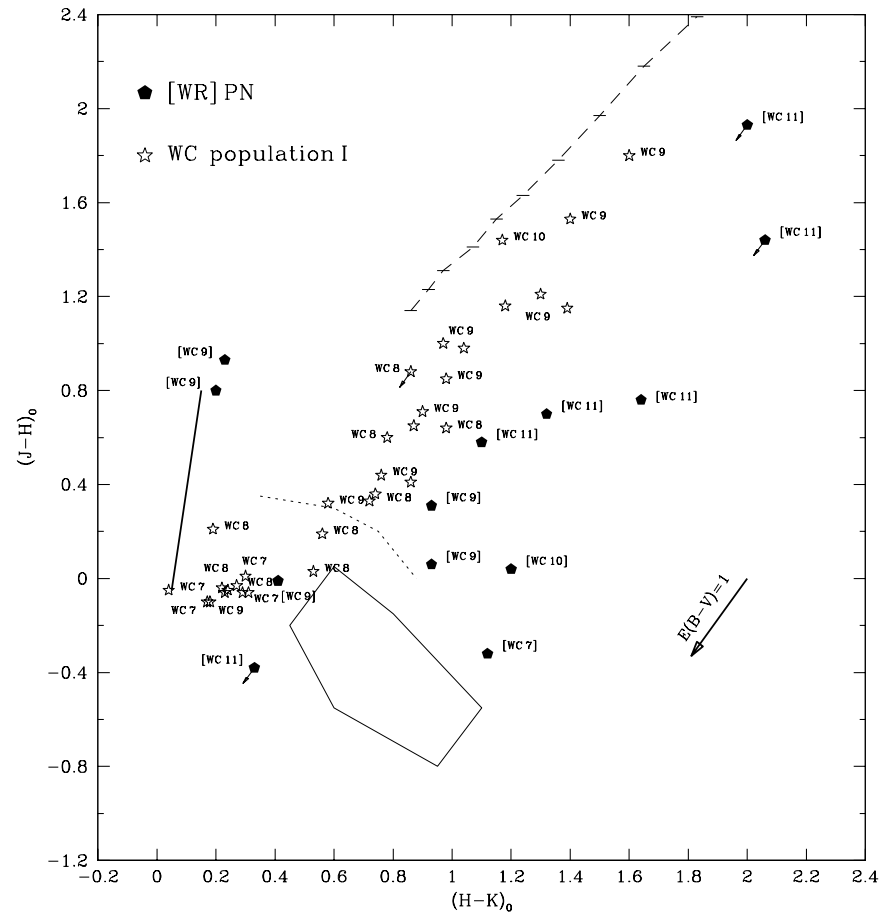

Fig. 2. Colour-colour diagram for late type [WR] PNN (pentagons) and population I WC stars (stars). For reference the same lines as in Fig. 1 are plotted.

features being the same), it is possible that the same mechanism of grain production and growth may be operating in both classes of objects. Recently Jones et al. (1999) reported two declines of the central star of He 3-1333 (CPD$56^{\circ} 8032$ ) resembling those observed in $\mathrm{R}$ Coronae Borealis variables and triggered by dust formation events. Note, however, that Nugis (1995) has argued that no dust is needed to interpret the near infrared spectra of population I Wolf-Rayet stars in most cases, and advocated for free-free and free-bound radiation of high density clumps in the stellar atmospheres. Whatever the interpretation may be (hot dust or free-free radiation from optically thick clumps) the shift to the right in the $(H-K)_{0}$ vs. $(J-H)_{0}$ plane from massive WC stars to [WR] PN can possibly be explained by a contribution of the nebular He I $2.058 \mu \mathrm{m}$ line in the $K$ band in the case of PN.

\section{Mid infrared photometry}

The mid-infrared radiation comes mostly from dust that was created during the AGB phase of the central star evolution. Therefore an analysis of this radiation can be extremely important for understanding the origin of the [WR] PN.

Figure 3 shows the colour dust temperature $T_{\mathrm{d}}$ of the planetary nebulae of our samples, as a function of the surface brightness in $\mathrm{H} \beta$. As in Fig. 1, [WR] PN are represented by pentagons, and non-[WR] PN by open circles. $T_{\mathrm{d}}$ was derived from the observed IRAS fluxes in the 25 and $60 \mu \mathrm{m}$ bands exactly in the same way as in Stasińska \& Szczerba (1999). Roughly the same trend is seen for the 


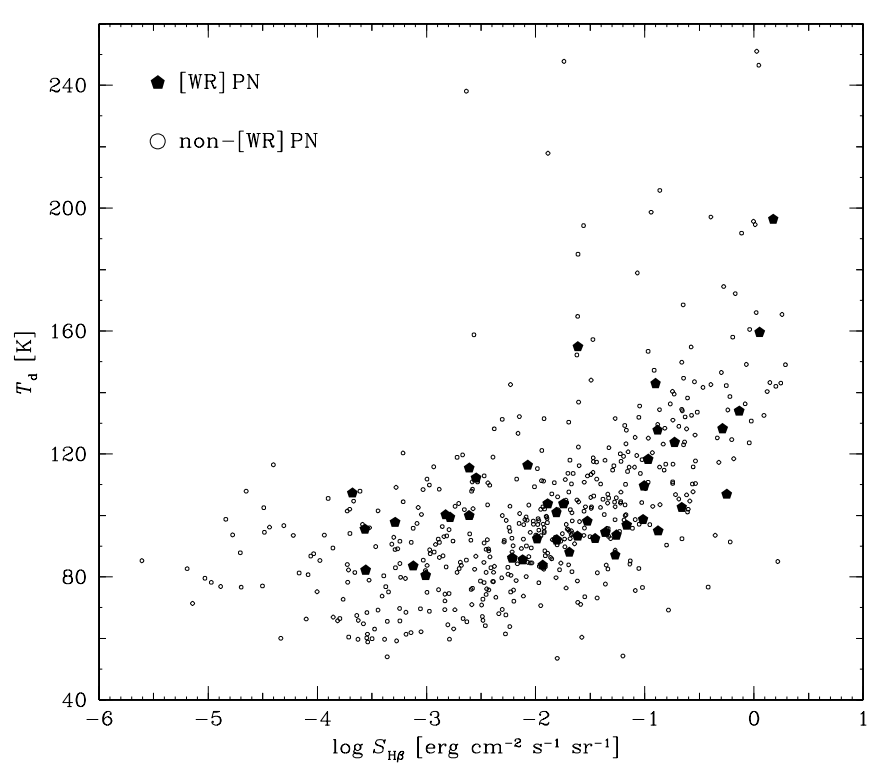

Fig. 3. The colour dust temperature $T_{\mathrm{d}}$ versus surface brightness in $\mathrm{H} \beta$. The same notation of symbols as in Fig. 1 .

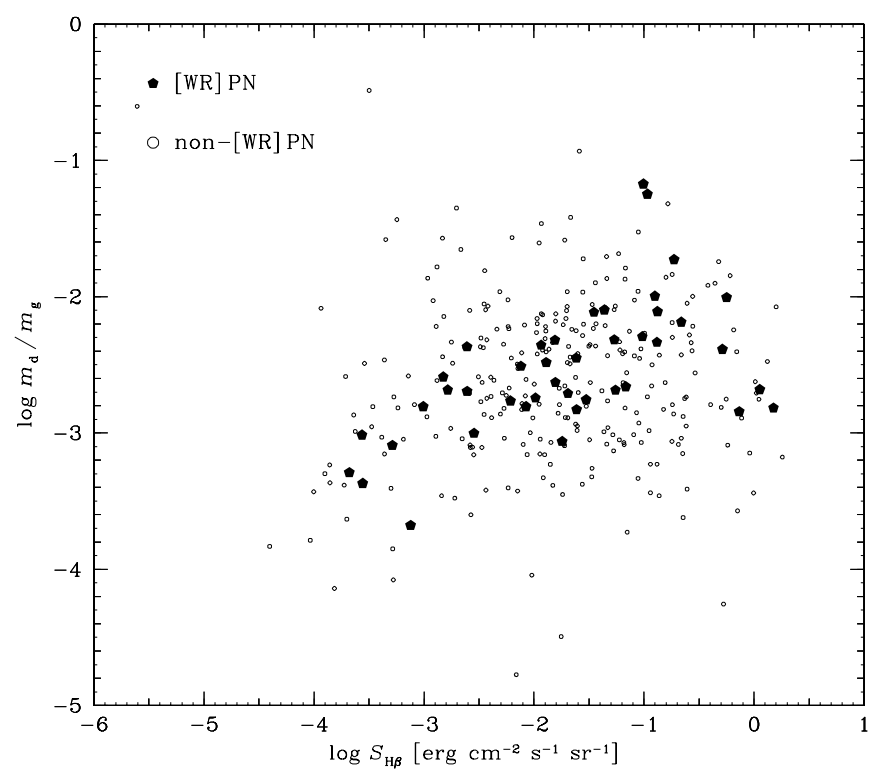

Fig. 4. The dust-to-gas mass ratio versus surface brightness in $\mathrm{H} \beta$. The same notation of symbols as in Fig. 1.

[WR] PN and non-[WR] PN samples. Such a trend is simply due to the fact that, as the planetary nebulae expand, the stellar radiation field becomes more diluted and the grains are less heated. From Table 1 one can also read that there is a tendency for $T_{\mathrm{d}}$ to decrease from late to early [WC] type (see also Fig. 6), which is consistent with the correlation seen between $S_{\mathrm{H} \beta}$ and the [WC] type (Górny \& Tylenda 2000; Peña et al. 2001). It is interesting to note however (Fig. 3) that $T_{\mathrm{d}}$ in [WR] PN levels off at about $80 \mathrm{~K}$ and never reaches the lowest values seen in some non-[WR] PN of same $S_{\mathrm{H} \beta}$. This may be due to either a different size distribution of the dust grains or perhaps reflects the fact that the $[\mathrm{WR}]$ phenomenon ceases before the end of the PN stage.

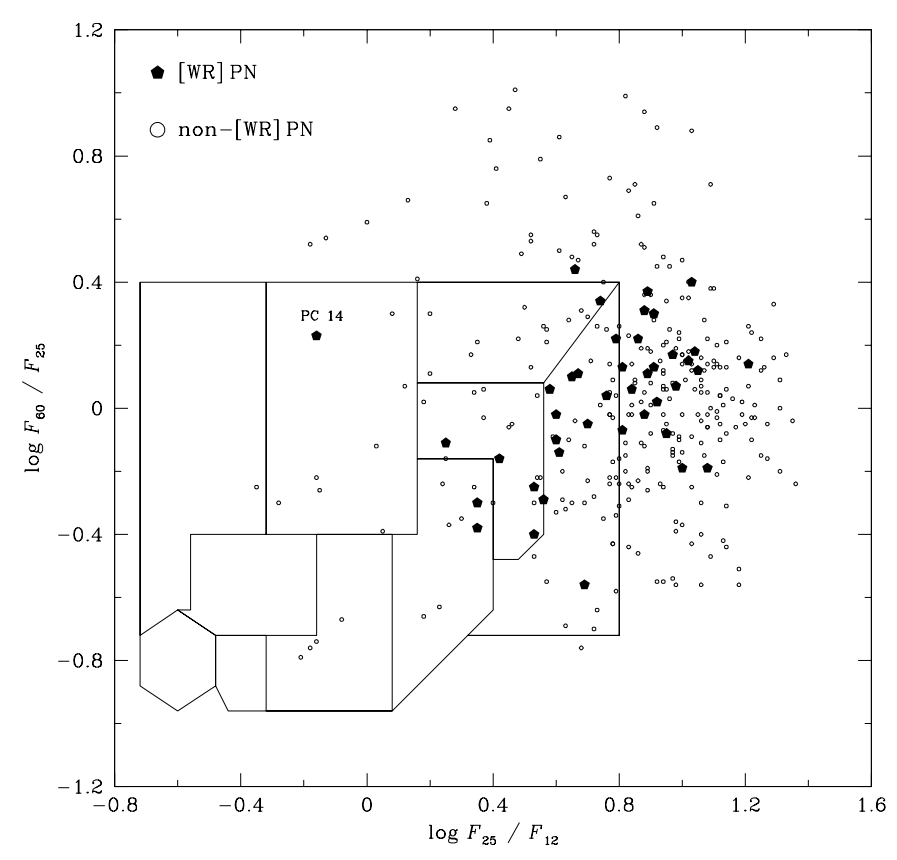

Fig. 5. The IRAS colour-colour diagram. Same meaning of symbols as in Fig. 1. The overplotted boxes occupied by different classes of objects come from van der Veen \& Habing (1988).

Another parameter worth examining is the dust-to-gas mass ratio, $m_{\mathrm{d}} / m_{\mathrm{g}}$ (see Fig. 4 ). This ratio has been derived following the method described in Stasińska \& Szczerba (1999). As far as the mean values of these parameters are concerned $\left(\log m_{\mathrm{d}} / m_{\mathrm{g}}\right.$ is $-2.43 \pm 0.85$ and $-2.59 \pm 0.62$ for [WR] PN non-[WR] PN, respectively) there is statistically no significant difference between the $[\mathrm{WR}] \mathrm{PN}$ and non-[WR] PN samples.

The non- $[\mathrm{WR}] \mathrm{PN}$ sample shows no correlation between $m_{\mathrm{d}} / m_{\mathrm{g}}$ and $S_{\mathrm{H} \beta}$ (see Stasińska \& Szczerba 1999). The Pearson correlation coefficient is 0.50 with an associated probability $p=0.41$ that the correlation is random. On the other hand, in the [WR] PN sample $m_{\mathrm{d}} / m_{\mathrm{g}}$ tends to decrease with decreasing $S_{\mathrm{H} \beta}$. The Pearson correlation coefficient is 0.57 for a sample of $42[\mathrm{WR}] \mathrm{PN}$ with $p=0.001$.

Note that the correlation seen in Fig. 4 does not necessarily mean that the actual dust-to-gas mass ratio decreases as the [WR] PN evolve. Stasińska \& Szczerba (1999) have shown that the method used to derive $m_{\mathrm{d}} / m_{\mathrm{g}}$ suffers from biases (essentially due to atomic emission line contamination) which mimic a decrease in $m_{\mathrm{d}} / m_{\mathrm{g}}$ with time. In fact, the [WR] PN in our Fig. 4 outline a similar slope to the one drawn by theoretical tracks in the constant $m_{\mathrm{d}} / m_{\mathrm{g}}$ case (see Fig. 8 in Stasińska \& Szczerba $1999)$. One way or the other, the $m_{\mathrm{d}} / m_{\mathrm{g}}-S_{\mathrm{H} \beta}$ correlation seen for [WR] PN in Fig. 4 suggests that these objects form a more homogeneous group than the rest of the $\mathrm{PN}$ population.

Figure 5 shows the IRAS colour-colour diagram with [WR] PN represented by pentagons and non-[WR]PN by open circles. Superimposed are the boxes defined by 


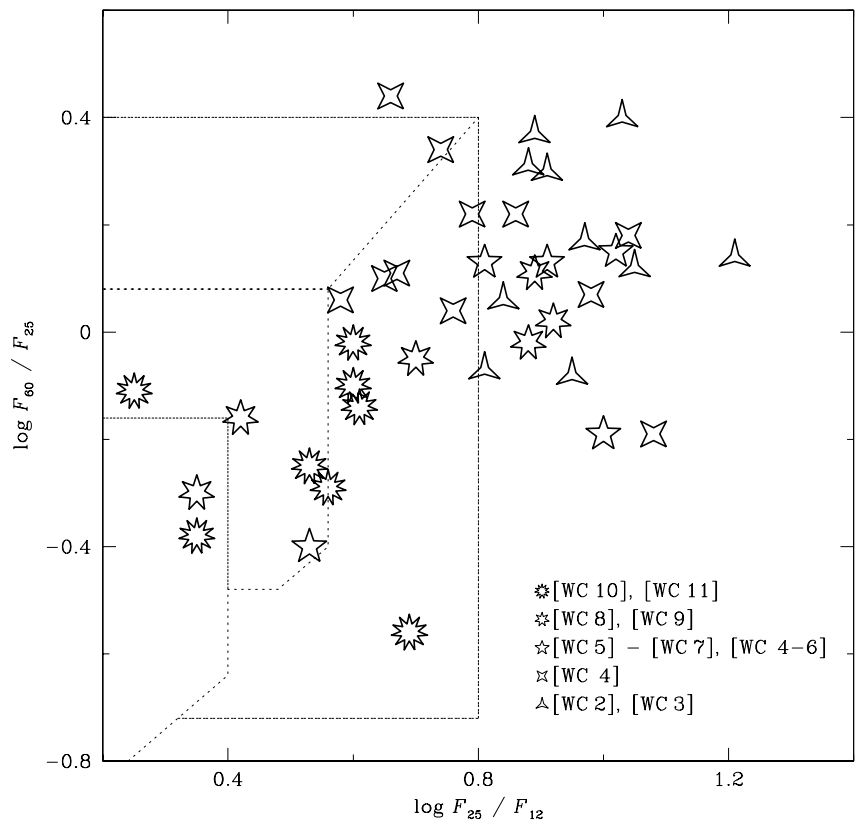

Fig. 6. The IRAS colour-colour diagram: a blow up of the region occupied by [WR] PN. Different symbols group [WR] PN according to the $[\mathrm{WC}]$ subtype of the central star.

van der Veen \& Habing (1988). It can be noticed that, in contrast to non-[WR] PN, planetary nebulae with [WR] central stars (with the exception of PC 14) occupy a very limited strip. The Pearson correlation coefficient in this diagram is 0.50 with an associated probability of 0.001 for the [WR] PN sample (excluding PC 14), while it is 0.02 with probability 0.69 for the non-[WR] PN sample. This means that the dust emission properties in [WR] PN are quite uniform, as compared to non-[WR] PN.

As shown by Fig. 6, which is an enlargement of the IRAS colour-colour diagram in the region occupied by PN, the strip occupied by [WR] PN seems to be a reflection of the evolutionary sequence of these objects. The different symbols in Fig. 6 mark different subsamples of [WR] PN grouped according to their [WC] subtype. One can see that going from later to earlier subtypes, i.e. from cooler to hotter central stars, "redder" IRAS colours are observed, indicating a cooling down of the dusty envelope. This cooling down is mainly due to the expansion of the nebula and the dilution of the heating radiation field, as already noted before.

\section{Mid infrared spectroscopy}

Analyzing the low resolution IRAS LRS spectra of planetary nebulae, Volk \& Cohen (1990) have defined 7 types of spectra: D, P, R, L, H, X and E. The first two classes represent objects with LRS spectra dominated by dust emission with features characteristic of either silicate or silicate-carbide (D) or carbon-based dust with structures attributed to PAH molecules (P). In R-type objects these emission structures disappear but dust continuum radiation still dominates the spectra. In L, H, X and E classes,

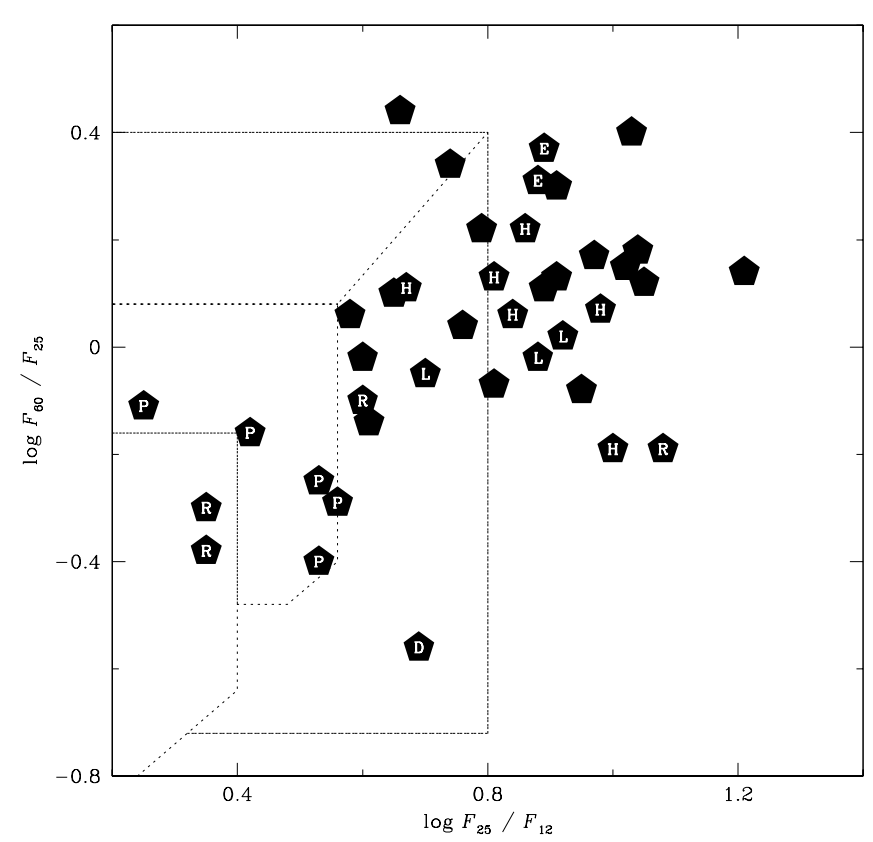

Fig. 7. The IRAS colour-colour diagram: a blow up of the region occupied by $[\mathrm{WR}] \mathrm{PN}$. The letters indicate the IRAS LRS spectral classes defined by Volk \& Cohen (1990).

dust is less and less important and the spectra are dominated by emission lines of gas ionized to higher and higher levels by the central star, whose temperature increases. The anticipated evolutionary sequence would be: $\mathrm{P}(\mathrm{D}) \rightarrow \mathrm{R} \rightarrow \mathrm{L} \rightarrow \mathrm{H} \rightarrow \mathrm{X} \rightarrow \mathrm{E}$. Unfortunately, because of a large scatter in colours, Volk \& Cohen (1990) were unable to identify this evolutionary sequence in their sample of 170 objects.

In Fig. 7 we have plotted the IRAS colour-colour diagram for the [WR] PN sample, marking the objects with the spectral classes defined by Volk \& Cohen (1990). One can clearly see a trend according to the expected evolutionary sequence. This strengthens our previous conclusions, namely that [WR] PN evolve from late to early [WC] and that they form a homogeneous group of objects in the PN population.

The distribution of planetary nebulae of different LRS spectral classes among [WR] PN and other PN is presented in Table 2. We see that the proportion of objects with detected $\mathrm{PAH}$ features (type $\mathrm{P}$ ) is much larger in the [WR] PN sample (24\%) than in the non-[WR] PN one $(8.5 \%)$.

Only true and probable PN analyzed and classified in Volk \& Cohen (1990) have been included in Table 2. With

Table 2. The distribution of planetary nebulae of different IRAS LRS spectral classes (Volk \& Cohen 1990) among $[\mathrm{WR}] \mathrm{PN}$ and other PN.

\begin{tabular}{lrrrrrrr}
\hline \hline & D & P & R & L & H & X & E \\
\hline$[$ WR] PN & 1 & 5 & 4 & 3 & 6 & - & 2 \\
non- $[\mathrm{WR}] \mathrm{PN}$ & 9 & 10 & 37 & 6 & 39 & 4 & 12 \\
\hline \hline
\end{tabular}




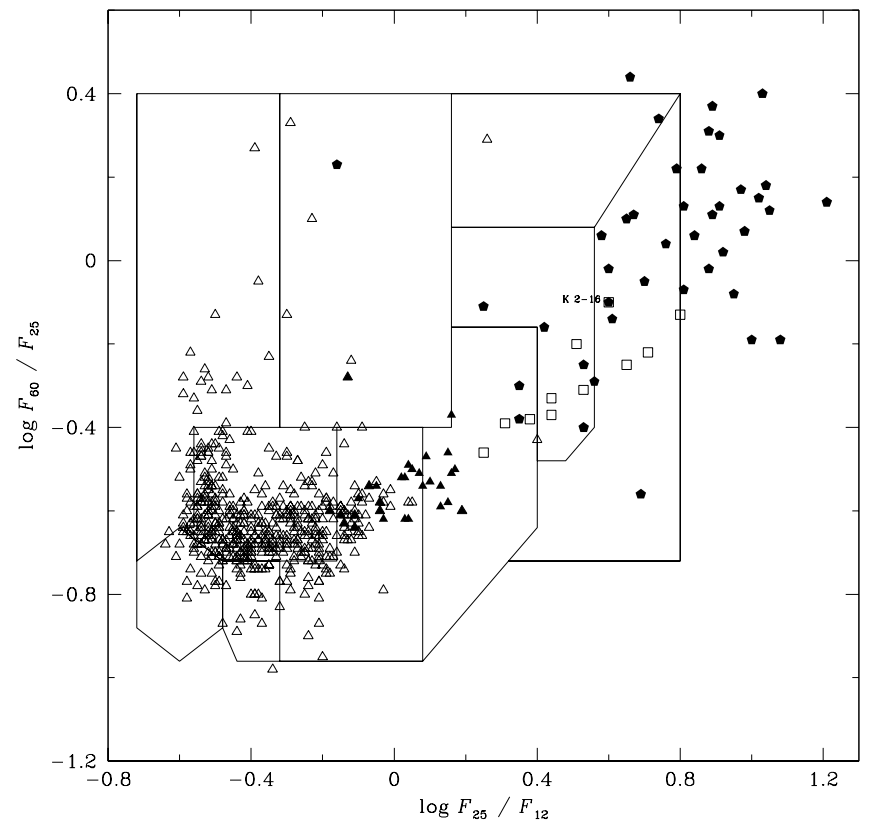

Fig. 8. The IRAS colour-colour diagram for [WR]PN and carbon-rich objects. Pentagons: [WR] PN; open triangles: classical carbon stars (Steffen et al. 1998); filled triangles: extreme carbon stars (Volk et al. 1992); open squares: transition objects (Volk et al. 1993).

new observations, the numbers of different categories are expected to increase. Indeed, a preliminary analysis of ISO SWS spectra of [WR] PN allowed us (Szczerba et al. 2001) to recognize signs of PAH emission in at least $75 \%$ of them.

One should notice that there is only one $\mathrm{D}$ type [WR] PN, namely the rather peculiar object SwSt 1 . Our ISO SWS spectrum confirms that amorphous silicate grain emission is present in this object but the spectrum is again dominated by strong PAH structures (Szczerba et al. 2001).

The presence of PAH molecules indicates that the predecessors of [WR] PN might be found among carbon AGB stars. This fact is in qualitative agreement with the mean $\mathrm{C} / \mathrm{O}$ abundance ratio of 1.45 found for [WR] $\mathrm{PN}$ in Górny \& Stasińska (1995). However, the mean C/O abundance for non-[WR] PN is not much different (1.26, see Górny \& Stasińska 1995). Taking these numbers at face value would imply that a carbon-rich environment is not a sufficient condition for the formation of PAHs and that specific conditions must prevail in [WR] PN to produce a much higher proportion of objects with PAH-dominated spectra than in the non-[WR] PN sample. However, the carbon abundance determinations in $\mathrm{PN}$ can be subject to important uncertainties (see e.g. Peña et al. 1998). A careful and systematic redetermination of carbon abundances in planetary nebulae, such as undertaken by Kwitter \& Henry (1996, 1998), would be very valuable.

The idea of a connection between [WR] PN and carbon AGB stars can be investigated by comparing the locations of both groups of objects in the IRAS colour-colour plane.

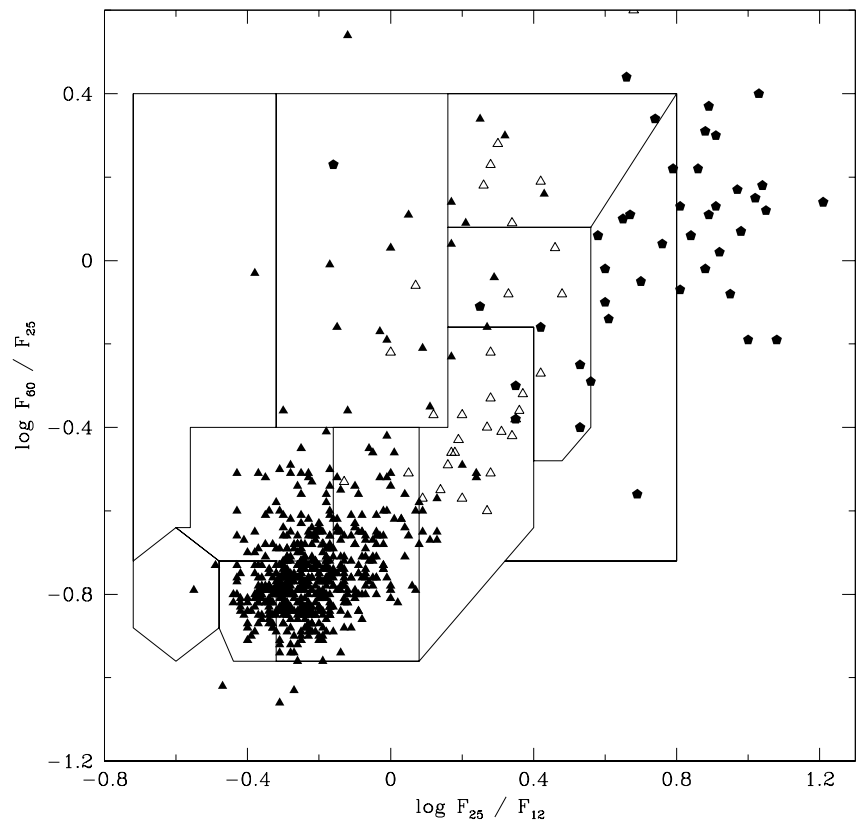

Fig. 9. The IRAS colour-colour diagram for [WR] PN and oxygen-rich objects. Pentagons: $[\mathrm{WR}] \mathrm{PN}$; filled triangles: AGB oxygen stars with silicate emission (Volk, private communication); open triangles: AGB oxygen stars with silicate absorption (Volk, private communication).

This is done in Fig. 8 where [WR] PN are represented by pentagons, classical carbon stars (Volk, private communication, see also Steffen et al. 1998) by open triangles and extreme carbon stars (Volk et al. 1992) by filled triangles. It is very tempting to conclude from this figure that the $[\mathrm{WR}] \mathrm{PN}$ sequence is a continuation of the carbon stars sequence. The link between both groups is set up by transition objects marked by open squares. They have been proposed as evolutionary descendants of carbon stars by Volk et al. (1993) basing on the similarity of LRS spectra. The status of these objects was unclear and these authors suspected that they are carbon rich proto-planetary or planetary nebulae. In fact one of these transition objects, IRAS 16416-2758, appeared to be a [WR] PN, i.e. K 2-16.

Figure 9 compares the locations of O-rich AGB stars and $[\mathrm{WR}] \mathrm{PN}$ in the IRAS colour-colour diagram. Here the link between the AGB stars and the [WR] PN is less evident than in Fig. 8. The oxygen-rich stars with silicate absorption, which are believed to be approaching the end of the AGB, do not seem to be directly followed by the $[\mathrm{WR}] \mathrm{PN}$.

It is worth noting, however, that model analyses of AGB and post-AGB evolution show that a similar or close position of objects in the IRAS colour-colour diagram does not necessarily imply that the objects are really in a similar stage of evolution or evolutionarity connected. Theoretical tracks in the IRAS colour-colour diagram are often very complicated and sensitively depend on many parameters, like chemical composition, mass loss characteristics, morphology and structure of the emitting dust (see e.g. van Hoof et al. 1997; Steffen et al. 1998; 
Szczerba \& Steffen 1998). In addition, in the case of evolved planetary nebulae, line emission from the ionized nebular gas can have an important influence on the photometric IRAS measurements (Leene \& Pottasch 1987; Stasińska \& Szczerba 1999).

The carbon-rich composition of the wind from the central stars, the presence of the PAH features, the nebular $\mathrm{C} / \mathrm{O}$ ratio typically well above 1 and the sequence observed in Fig. 8 are strong arguments in favour of the idea that the [WR] PN evolve from the carbon AGB stars. However, recent observations with ISO show that the situation is not as simple as that. Waters et al. (1998), in the spectra of $\mathrm{He} 2-113$ and $\mathrm{BD}+30^{\circ} 3639$, apart from strong $\mathrm{PAH}$ features (signs of a carbon nature of these objects) have found features produced by crystalline olivines and pyroxenes which are fingerprints of oxygenrich matter. The same properties have been observed in He 3-1333 (Cohen et al. 1999) and thanks to our own ISO observations we can supplement this group with two more [WR] PN: M 2-43 and He 2-459. In addition, maser $\mathrm{OH}$ emission has been observed in Vo 1, He 2-142 and again $\mathrm{BD}+30^{\circ} 3639$ (see Chen et al. 2001 and references therein). Thus there is evidence for O-rich material in many [WR] PN.

There are several possible explanations for a simultaneous presence of oxygen- and carbon-rich dust around some [WR] PN (Cohen et al. 1999). One of them is a very recent change of the surface composition in the progenitors of $[\mathrm{WR}]$ type stars. Whatever the reason for this change might be, the evolution of these objects would actually follow from the O-rich AGB stars. The sequence observed in Fig. 8 would then only mean that the general dust properties in carbon stars and [WR] PN are similar.

\section{Summary and conclusions}

We have analyzed the near and mid infrared photometric and spectroscopic properties of Galactic planetary nebulae, sorting out the [WR] PN in order to get some insight into their dust properties and, consequently, their evolutionary status. This work is a follow-up of previous works, based on optical data, aimed at understanding the nature and evolution of [WR] PN (Górny \& Stasińska 1995; Acker et al. 1996; Górny \& Tylenda 2000; Peña et al. 2001).

Our main conclusions are the following.

In the $(H-K)_{0}$ versus $(J-H)_{0}$ diagram, [WR] PN behave quite differently from non- $[\mathrm{WR}] \mathrm{PN}$ with the latter being mostly confined to the "nebular box" defined by Whitelock (1985). Some [WR] PN that lie close to the stellar main sequence region might have binary central stars, although contamination of the photometric fluxes by field stars is also a likely explanation. A sizeable proportion of [WR] PN with late type central stars seems to contain hot dust (1000-2000K), which could have be formed in the winds of the stars, similarly to what is proposed for massive, population I late-type WC stars.

The mean dust temperature is shown to decrease as the nebular surface brightness in $\mathrm{H} \beta$ decreases, in a similar fashion between [WR] PN and non-[WR] PN. For $[\mathrm{WR}] \mathrm{PN}$, it decreases with decreasing [WC] spectral type, giving an additional indication that the $[\mathrm{WC}]$ sequence is an evolutionary sequence from late to early-type, as suggested by nebular studies based on optical data (Górny \& Tylenda 2000; but see also Peña et al. 2001). The [WR] PN do not reach the lowest dust temperatures seen in non[WR] PN of the same surface brightness. This probably results from the fact that $[\mathrm{WR}]$ stars cease their wind activity while the surrounding nebulae are still visible.

In diagrams showing $m_{\mathrm{d}} / m_{\mathrm{g}}$ as a function of $S_{\mathrm{H} \beta}$, $[\mathrm{WR}] \mathrm{PN}$ stand out with respect to non-[WR] PN. They show a significant correlation between $m_{\mathrm{d}} / m_{\mathrm{g}}$ and $S_{\mathrm{H} \beta}$, while no correlation is seen for the remaining PN. This does not necessarily mean that the true dust-to-gas mass ratio decreases with time. The methods used to derive $m_{\mathrm{d}} / m_{\mathrm{g}}$ are biased due to the increasing importance of atomic emission lines in old nebulae (see Stasińska \& Szczerba 1999). The observed correlation indicates, however, that [WR] PN form a homogeneous class of objects in which the dust properties are quite uniform with respect to $\mathrm{PN}$ in general.

The uniformity of the dust properties of $[\mathrm{WR}] \mathrm{PN}$ is confirmed by the fact that these objects form a wellconfined sequence in the IRAS colour-colour diagram. This sequence and an analysis of the LRS IRAS spectra confirm the general evolution of the $[\mathrm{WR}] \mathrm{PN}$ from late to early [WC] types.

The IRAS spectroscopic properties of [WR] PN, when considered together with those of carbon stars and transition objects, seem to indicate an evolutionary sequence from carbon AGB stars to [WR] PN, or at least a similarity in their dust properties.

Many PN with cool [WR] central stars show the presence of oxygen-rich material, suggesting that their progenitors are not carbon stars. On the other hand, the overwhelming proportion of $[\mathrm{WR}] \mathrm{PN}$ showing PAH features argues that at least some dust was formed in a carbonrich environment.

Acknowledgements. The authors acknowledge support from grants No. 2.P03D.002.13 \& No. 2.P03D.020.17 of the Polish Committee for Scientific Research and from the Jumelage France-Pologne.

\section{References}

Acker, A., Ochsenbein, F., Stenholm, B., et al. 1992, Strasbourg-ESO Catalogue of Galactic Planetary Nebulae, European Southern Observatory, Garching

Acker, A., Górny, S. K., \& Cuisinier, F. 1996, A\&A, 305, 944

Acker, A., Parthasarathy, M., \& Stenholm, B. 1999, Quantitative classification of [WC] nuclei of planetary nebulae, preprint

Allen, D. A., \& Glass, I. S. 1974, MNRAS, 167, 337

Chen, P. S., Szczerba, R., Kwok, S., \& Volk, K. 2001, A\&A, 368,1006 
Cherchneff, I., \& Tielens, A. G. G. M. 1995, in Wolf-Rayet Stars: Binaries, Colliding Wind, Evolution, ed. K. A. van der Hucht, \& P. M. Williams (Kluwer, Dordrecht), Proc. IAU Symp., 163, 346

Ciardullo, E., Bond, H. E., Sipior, et al. 1999, AJ, 118, 488

Cohen, M., Barlow, M. J., Sylvester, R. J., et al. 1999, ApJ, 513, L135

De Marco, O., Barlow, M. J., \& Storey, P. J. 1997, MNRAS, 292,86

Fouqué, P., Le Bertre, T., Epchtein, N., Guglielmo, F., \& Kerschbaum, F. 1992, A\&AS, 93, 151

Garcia-Lario, P., Manchado, A., Suso, S. R., Pottasch, S. R., \& Olling, R. 1990, A\&AS, 82, 497

Garcia-Lario, P., Manchado, A., Pych, W., \& Pottasch, S. R. 1997, A\&AS, 126, 479

Gaylard, M. J., West, M. E., Whitelock, P. A., \& Cohen, R. J. 1989, MNRAS, 236, 247

Gezari, D. Y., Pitts, P. S., \& Schmitz, M. 1997, Catalog of Infrared Observations, Edition 4, unpublished

Górny, S. K. 2001, Ap\&SS, 275, 67

Górny, S. K., \& Stasińska, G. 1995, A\&A, 284, 949

Górny, S. K., \& Stasińska, G. 1996, in Hydrogen-Deficient Stars, ed. C. S. Jeffery, \& U. Heber, ASP Conf. Ser., 96, 209

Górny, S. K., \& Tylenda, R. 2000, A\&A, 362, 1008

Hamann, W.-R. 1997, in Planetary Nebulae, ed. H. J. Habing, \& H. J. G. L. M. Lamers (Kluwer), IAU Symp., 180, 91

Hu, J. Y., \& Dong, Y. S. 1992, Chin. Sci. Bull., 37, 213

Hu, J. Y., Slijkhuis, S., de Jong, T., \& Jiang, B. W. 1993, A\&AS, 100, 413

Iben, I., Kaler, J. B., Truran, J. W., \& Renzini, A. 1983, ApJ, 264, 605

Jones, A., Lawson, W., \& De Marco, O. 1999, The Observatory, 119,76

Koesterke, L., \& Hamann, W.-R. 1997a, A\&A, 320, 91

Koesterke, L., \& Hamann, W.-R. 1997b, in Planetary Nebulae, ed. H. J. Habing, \& H. J. G. L. M. Lamers (Kluwer), IAU Symp., 180, 114

Koesterke, L., Dreizler, S., \& Rauch, T. 1998, A\&A, 330, 1041

Koorneef, J. 1983, A\&AS, 51, 489

Kwitter, K. B., \& Henry, R. B. C. 1996, ApJ, 473, 304

Kwitter, K. B., \& Henry, R. B. C. 1998, ApJ, 493, 247

Le Bertre, T., Heydari-Malayeri, M., Epchtein, N., Gouiffes, C., \& Perrier, C. 1989, A\&A, 225, 417

Leene, A., \& Pottasch, S. R. 1987, in Planetary and ProtoPlanetary Nebulae: From IRAS to ISO, ed. A. PreiteMartinez (Reidel), 233

Leuenhagen, U., \& Hamann, W.-R. 1994, A\&A, 283, 567

Leuenhagen, U., \& Hamann, W.-R. 1998, A\&A, 330, 265

Leuenhagen, U., Hamann, W.-R., \& Jeffery, C. S. 1996, A\&A, 312,167
Nugis, T. 1995, in Wolf-Rayet Stars: Binaries, Colliding Wind, Evolution, ed. K. A. van der Hucht, \& P. M. Williams (Kluwer, Dordrecht), Proc. IAU Symp., 163, 371

Peña, M., \& Torres Peimbert, S. 1987, RevMexAA, 14, 534

Peña, M., Stasińska, G., Esteban, C., et al. 1998, A\&A, 337, 866

Peña, M., Stasińska, G., \& Medina, S. 2001, A\&A, 367, 983

Perinotto, M. 1993, in Planetary Nebulae, ed. A. Acker, \& R. Weinberger (Kluwer), 57

Persi, P., Preite-Martinez, A., Ferrari-Toniolo, M., \& Spinoglio, L. 1987, in Planetary and Proto-Planetary Nebulae: From IRAS to ISO, ed. A. Preite-Martinez (Reidel), 221

Preite-Martinez, A., \& Persi, P. 1989, A\&A, 218, 264

Phillips, J. P., \& Cuesta, L. 1994, A\&AS, 104, 169

Rieke, H. H., \& Lebofsky, M. J. 1985, ApJ, 288, 618

Schönberner, D., \& Blöcker, T. 1992, in Atmospheres of Early Type Stars, ed. U. Heber, \& C. S. Jeffery (Springer), 305

Stasińska, G., \& Szczerba, R. 1999, A\&A, 352, 297

Steffen, M., Szczerba, R., \& Schönberner, D. 1998, A\&A, 337, 149

Szczerba, R., \& Steffen, M. 1998, Ap\&SS, 262, 187

Szczerba, R., Górny, S. K., Stasińska, G., Siódmiak, N., \& Tylenda, R. 2001, Ap\&SS, 275, 113

Tylenda, R., Acker, A., \& Stenholm, B. 1993, A\&AS, 102, 595

Tylenda, R., \& Górny, S. K. 1993, in Bachotek Workshop, Evolution of Planetary Nebula Nuclei, ed. R. Tylenda, Acta Astr., 43, 389

van der Hucht, K. A., Conti, P. S., Lundström, I., \& Stenholm, B. 1981, Space Sci. Rev., 28, 227

van der Veen, W. E. C. J., \& Habing, H. J. 1988, A\&A, 194, 125

van der Veen, W. E. C. J., Habing, H. J., \& Geballe, T. R. 1989, A\&A, 226, 108

van Hoof, P. A. M., Oudmeijer, R. D., \& Waters, L. B. F. M. 1997, MNRAS, 289, 371

Volk, K., \& Cohen, M. 1990, AJ, 100, 485

Volk, K., Kwok, S., \& Langill, P. L. 1992, ApJ, 391, 285

Volk, K., Kwok, S., \& Woodsworth, A. W. 1993, ApJ, 402, 292

Waters, L. B. F. M., Beintema, D. A., Zijlstra, A. A., et al. 1998, A\&A, 331, L61

Webster, L. B., \& Glass, I. S. 1974, MNRAS, 166, 491

Williams, P. M., van der Hucht, K. A., \& Thé, P. S. 1987, A\&A, 182, 91

Whitelock, P. A. 1985, MNRAS, 213, 59

Zijlstra, A. A., Gaylard, M. J., Te Lintel Hekkert, P., et al. 1991, A\&A, 243, L9

Zhang, C. Y., \& Kwok, S. 1990, A\&A, 237, 479

Zubko, V. G. 1998, MNRAS, 295, 109 